\title{
Finite Difference Method for Boundary Value Problems in Ordinary Differential Equations
}

\author{
Biruk Endeshaw \\ Department of Mathematics, Wolaita Sodo University, PO box 138, Wolaita Sodo, Ethiopia \\ E-mail: birukendeshaw@gmail.com
}

\begin{abstract}
In this paper, a second order numerical method based on finite difference method with uniform mesh is presented for solving boundary value problems. Numerical solution is found for the boundary value problem using finite difference method and the results are tabulated and compared with analytical solution. Graphs are also depicted in support of the numerical results. The present method is simple and it approximates the exact solution very well.
\end{abstract}

Keywords: Boundary value problem, Finite Difference Method, Ordinary Differential Equations

DOI: $10.7176 /$ MTM/9-10-03

Publication date: October $31^{\text {st }} 2019$

\section{Introduction}

Ordinary and partial differential equation have long played important roles in the history of modelling physical phenomena; they continue to serve as indispensable tools in future investigations. In the theory and application of ordinary and partial differential equations, one may be interested to find a solution to a differential equation satisfying certain defined conditions. If the conditions are given at only one point of the independent variable, we have initial conditions; where as if the conditions are given at more than one point of the independent variable, they are called boundary conditions (BC). The problem of finding a solution of an $n^{\text {th }}$ order differential equation together with n-initial conditions is called an initial value problem (IVB), however if the n-boundary conditions are considered, the problem is called boundary value problem (BVP).

Boundary value problems for linear second order equations are particularly important because of numerous applications in science and technology. In this paper as in most physical applications, boundary conditions are always imposed at end points of an interval.

In this paper, we consider the following second order boundary value problem

$$
-\frac{d}{d x}\left(p(x) \frac{d y}{d x}\right)+q(x) y=f(x), a<x<b,
$$

with the boundary conditions

$$
y(a)=y_{a} \text { and } y(b)=y_{b}
$$

where $y_{a}$ and $y_{b}$ are given real numbers.

While there are many numerical methods for solving such boundary value problems, the method of finite difference is most commonly used. The basis of the finite difference method is the replacement of all derivatives occurring by the corresponding difference quotients; this is applicable to any problem in differential equations and the resulting linear system of equation is solved by any standard procedure. These roots are the value of the required solution at the pivotal points.

Some researchers have studied and numerically solved second order boundary-value problems using different method with different boundary conditions, for instance, the collocation method [2], finite difference method [9], shooting method [1], quantic spline [14], non-polynomial spline [6] and references therein. Hence, the purpose of this paper is to develop a numerical method for solving second order boundary-value problem (1).

This paper is arranged in the following manner. In section 2, some definitions and notions are presented. In section 3 , we formulate and described the proposed method. We have discussed convergence of the proposed method under appropriate condition in Section 4. The Numerical examples and applications of linear boundary value problems of the second order are discussed in Section 5 and Section 6, respectively. Finally, conclusion drawn is presented.

\section{Preliminaries}

In this section, some of the basic ideas, definitions and theorems which are relevant to the solution methods of 
finite difference in second order linear boundary value problem of ordinary differential equation.

Definition 2.1. An equation containing the derivative of one or more dependent variables with respect to one or more independent variables is said to be differential equation.

Definition 2.2. A differential equation is said to be an ordinary differential equation if it contains only ordinary derivatives of one or more dependent variables with respect to a single independent variable.

Definition 2.3. An $n^{\text {th }}$ order ordinary differential equation is said to be linear in $y$ if it can be written in the form

$$
a_{n}(x) y^{(n)}+a_{n-1}(x) y^{(n-1)}+\cdots+a_{0}(x) y=f(x)
$$

Where $a_{n}(x)$ are continuous function

\subsection{Linear boundary value problem}

The differential equation and the boundary condition are linear (these are called BVP) and for which the boundary conditions are based on only two points say $x_{1}=a$ and $x_{2}=b$ where $a<b$. The differential equation may be written in the form

$$
a_{n}(x) y^{(n)}+a_{n-1}(x) y^{(n-1)}+\cdots+a_{0}(x) y=f(x)
$$

With boundary conditions

$$
y\left(x_{1}\right)=a, y\left(x_{2}\right)=b
$$

The differential equation is homogeneous when $f(x)=0$ in $[a, b]$ otherwise non-homogeneous. The boundary value problem is called homogeneous when the differential and all the boundary condition are homogeneous. That is

$$
a_{n}(x) y^{(n)}+a_{n-1}(x) y^{(n-1)}+\cdots+a_{0}(x) y=f(x)
$$

with boundary conditions $y\left(x_{1}\right)=y\left(x_{2}\right)=\alpha$.

Definition 2.4. A set of function $f_{1}(x), f_{2}(x), \ldots, f_{n}(x)$ is said to be linearly independent on an interval $I$ if the only constants for which $\alpha_{1} f_{1}(x)+\alpha_{2} f_{2}(x)+\cdots+\alpha_{n} f_{n}(x)$ for every $x$ in the interval $I$ are $\alpha_{1}=\alpha_{2}=\cdots=\alpha_{n}=0$.

Theorem 2.1. Let $y_{1}, y_{2}, \ldots, y_{n}$ be the solution of equation (4) on an interval $I$, then the linear combination $y=$ $\alpha_{1} y_{1}+\alpha_{2} y_{2}+\cdots+\alpha_{n} y_{n}$ where $\alpha_{i}$, for $i=1,2, \ldots, \mathrm{n}$ are arbitrary constants is also a solution of equation (4).

2.2. Linear system of equations

A number of problems in numerical analysis can be reduced to system of linear equations. Let us consider a system of real linear equations, which may be written in matrix form

$$
A x=r
$$

Where, $A=\left(a_{j k}\right)$ is the $n \times n$ matrix of coefficients, $x=\left(x_{j}\right)$ the column matrix of the unknowns and, $r=\left(r_{i}\right)$ the column matrix of the right-hand sides.

Definition 2.5. A matrix $A$ is called irreducible if there is no permutation matrix $P$ such that $P^{T} A P$ has the form

$$
P^{T} A P=\left[\begin{array}{cc}
A_{11} & A_{12} \\
0 & A_{22}
\end{array}\right]
$$

Where $A_{11}$ is a $P \times P$ matrix and $A_{22}$ a $q \times q$ matrix with $p+q=n, p>0, q>0$.

Definition 2.6. A matrix $A$ is said to be diagonally dominant if for every row of the matrix, the magnitude of the diagonal entry in a row is larger than or equal to the sum of the magnitudes of all the other (non-diagonal) entries in that row. More precisely, the matrix $A$ is diagonally dominant if

$$
\left|a_{i i}\right| \geq \sum_{j=1, i \neq j}^{n}\left|a_{i j}\right|
$$

Where $a_{i j}$ denotes the entry in the $i^{\text {th }}$ row and $j^{\text {th }}$ column.

Note that this definition uses a weak inequality, and is therefore sometimes called weak diagonal dominance. If a strict inequality $(>)$ is used, this is called strict diagonal dominance. The unqualified term diagonal dominance can mean both strict and weak diagonal dominance.

Theorem 2.2. Assume the matrix $A=\left(a_{j k}\right)$ is irreducible and weakly row-diagonally dominant that is $A$ is rowdiagonally dominant

$$
\sum_{k=1, k \neq j}^{n}\left|a_{j k}\right| \leq\left|a_{j j}\right|, \quad j=1,2, \ldots, n
$$


With inequality holding for at least one row $j$, then the Jacobi iterations converge for each $y, x$ when applied to the equation $A X=Y$.

\subsection{The Finite Difference Method for Linear BVP in ODEs}

The solution of BVP by finite difference method is accomplished by the following steps.

1. Discretizing the continuous solution domain into a discrete finite difference grid.

2. Approximating the exact derivatives in the Ordinary Differential Equation by finite difference approximation.

3. Substituting finite difference approximation into the Ordinary Differential Equation to obtain algebraic finite difference equation.

4. Solving the resulting system of equations by any standard procedure.

\section{Description of the Method}

We first consider the case of a linear two-point boundary value problem of the form

$$
L[y] \equiv-\frac{d}{d x}\left(p(x) \frac{d y}{d x}\right)+q(x) y=f(x), y(a)=y_{a} \text { and } y(b)=y_{b}
$$

Where we assume that $p(x) \geq 0$ and $q(x) \geq 0$. In order to develop the numerical method for finding the solution of differential equation (6), the interval $[a, b]$ is divided into equal $n$ subintervals using the mesh points $x_{j}=a+$ $j h, j=0,1, \ldots, N, x_{0}=a, x_{n}=b$, and $h=(b-a) / N$.

Consider the special case when $p(x)$ is constant. Applying Taylor series expansions on $y(x-h)$ and $y(x+h)$ respectively and adding the two, we obtain second order central difference

$$
\frac{y(x+h)-2 y(x)+y(x-h)}{h^{2}}=y^{\prime \prime}(x)+\frac{h^{2}}{12} y^{(4)}(\xi), \quad \xi \in[x-h, x+h]
$$

If we apply the above equation (7) at the point $x=x_{j}$ and insert it into the differential equation (6), we get

$$
\begin{gathered}
0=-p y^{\prime \prime}\left(x_{j}\right)+q\left(x_{j}\right) y\left(x_{j}\right)-f\left(x_{j}\right) \\
L y\left(x_{j}\right) \equiv 0=-p \frac{\left[y\left(x_{j+1}\right)-2 y\left(x_{j}\right)+y\left(x_{j-1}\right)\right]}{h^{2}}+q\left(x_{j}\right) y\left(x_{j}\right)-f\left(x_{j}\right)+p\left[\frac{h^{2}}{12} y^{(4)}(\xi)\right]
\end{gathered}
$$

We then define an approximation $y_{j}$ to $y\left(x_{j}\right)$ by omitting the $O\left(h^{2}\right)$ error terms. We let $y_{j}, j=1,2, \ldots, N-1$ satisfy:

$$
L_{h} y_{j} \equiv p \frac{\left[-y_{j+1}+2 y_{j}-y_{j-1}\right]}{h^{2}}+q\left(x_{j}\right) y_{j}=f\left(x_{j}\right)
$$

Since the boundary conditions are given, we set $y_{0}=y_{a}$ and $y_{N}=y_{b}$. Hence, we have $N-1$ linear equations for the $N-1$ unknowns $y_{1}, \ldots, y_{N-1}$.

For later use, it is useful to define the local truncation error of the method from Eqs. (8) and (9), given by

$$
\tau_{j}=L_{h}\left(x_{j}\right)-L y\left(x_{j}\right)
$$

This is basically how well the true solution satisfies the discrete equations. Using the Taylor expansions defined above, we see that

$$
\tau_{j}=L_{h}\left(x_{j}\right)-L y\left(x_{j}\right)=-p\left[\frac{h^{2}}{12} y^{(4)}(\xi)\right]
$$

We next consider the approximate discrete system in more detail. Multiplying our discrete equation (9) by $h^{2}$ and collecting terms, we get

$$
-p y_{j+1}+\left[2 p+h^{2} q\left(x_{j}\right)\right] y_{j}-p y_{j-1}=h^{2} f\left(x_{j}\right)
$$

Setting $a_{j}=2 p+h^{2} q\left(x_{j}\right)$, we may write this system of linear equations in matrix form as: 


$$
\left(\begin{array}{cccccc}
a_{1} & -p & 0 & 0 & \ldots & 0 \\
-p & a_{2} & -p & 0 & \ldots & 0 \\
\cdot & \cdot & \cdot & \cdot & \cdot & \cdot \\
\cdot & \cdot & \cdot & \cdot & \cdot & \cdot \\
0 & \cdot & & -p & a_{N-2} & -p \\
0 & \cdot & & & -p & a_{N-1}
\end{array}\right)\left[\begin{array}{c}
y_{1} \\
y_{2} \\
\cdot \\
\cdot \\
y_{N-2} \\
y_{N-1}
\end{array}\right]=h^{2}\left[\begin{array}{c}
f\left(x_{1}\right) \\
f\left(x_{2}\right) \\
\cdot \\
\cdot \\
f\left(x_{N-2}\right) \\
f\left(x_{N-1}\right)
\end{array}\right]+\left[\begin{array}{c}
p y_{a} \\
0 \\
\cdot \\
\cdot \\
0 \\
p y_{b}
\end{array}\right]
$$

Thus, we need to solve the linear system $A y=F$, where $A$ is a symmetric tri-diagonal matrix. Note that if $\min _{[a, b]} q(x) \geq q^{*}>0$, then $A$ is strictly diagonally dominant and hence invertible.

Hence the Jacobi iteration of linear system of equation converges and by theorem (2.2) converges for each $y, f$ when applied to $A y=F$.

Now let us return to the case when $p$ depends on $x$. One possibility is to write

$$
L y=-p y^{\prime \prime}-p^{\prime} y^{\prime}+q y
$$

and approximate the first term at $x=x_{j}$ (as above) by

$$
p\left(x_{j}\right) \frac{\left[-y_{j+1}+2 y_{j}-y_{j-1}\right]}{h^{2}}
$$

To get an approximation to $y^{\prime}$ that is also second order, we could use the Taylor series to write

$$
\frac{y(x+h)-y(x-h)}{2 h}=y^{\prime}(x)+\frac{h^{3}}{6} y^{\prime \prime \prime}(\eta)
$$

for some $\eta \in[x-h, x+h]$.

In that case, our discrete equation (10) would become

$$
-\left[p\left(x_{j}\right)-\frac{h p^{\prime}\left(x_{j}\right)}{2}\right] \mathrm{y}_{\mathrm{j}-1}+\left[2 p\left(x_{j}\right)+h^{2} q\left(x_{j}\right)\right] \mathrm{y}_{\mathrm{j}}-\left[p\left(x_{j}\right)+\frac{h p^{\prime}\left(x_{j}\right)}{2}\right] y_{j+1}=h^{2} f\left(x_{j}\right)
$$

There are two drawbacks to this approach. First, the matrix we get will not be symmetric, and thus will involve more work to solve. To see this, let

$$
a_{j}=2 p\left(x_{j}\right)+h^{2} q\left(x_{j}\right), \quad b_{j}=p\left(x_{j}\right)-\frac{h p^{\prime}\left(x_{j}\right)}{2}, \quad c_{j}=p\left(x_{j}\right)-\frac{h p^{\prime}\left(x_{j}\right)}{2}
$$

Then, we may write this system of linear equations in matrix form as:

$$
\left(\begin{array}{cccccc}
a_{1} & -c_{1} & 0 & 0 & \ldots & 0 \\
-b_{2} & a_{2} & -c_{2} & 0 & \ldots & 0 \\
\cdot & \cdot & \cdot & \cdot & \cdot & \cdot \\
\cdot & \cdot & \cdot & \cdot & \cdot & \cdot \\
0 & \cdot & & -b_{N-2} & a_{N-2} & -c_{N-2} \\
0 & \cdot & & & -b_{N-2} & a_{N-1}
\end{array}\right)\left[\begin{array}{c}
y_{1} \\
y_{2} \\
\cdot \\
\cdot \\
y_{N-2} \\
y_{N-1}
\end{array}\right]=h^{2}\left[\begin{array}{c}
f\left(x_{1}\right) \\
f\left(x_{2}\right) \\
\cdot \\
\cdot \\
f\left(x_{N-2}\right) \\
f\left(x_{N-1}\right)
\end{array}\right]+\left[\begin{array}{c}
b_{1} y_{a} \\
0 \\
\cdot \\
\cdot \\
0 \\
c_{N-1} y_{b}
\end{array}\right]
$$

When $p$ is not constant, we do not expect that $b_{j+1}=c_{j}$. Another drawback to this approach is that we would also have to compute $p^{\prime}$.

Instead, we consider another way to approximate the derivatives in the equation. Using Taylor series expansions, we get either

$$
\begin{aligned}
& v(x \pm \epsilon)=v(x) \pm \epsilon v^{\prime}(x)+\frac{\epsilon^{2}}{2} v^{\prime \prime}(x) \pm O\left(\epsilon^{3}\right) \text { or } \\
& v(x \pm \epsilon)=v(x) \pm \epsilon v^{\prime}(x)+\frac{\epsilon^{2}}{2} v^{\prime \prime}(x) \pm \frac{\epsilon^{3}}{6} v^{\prime \prime \prime}(x)+O\left(\epsilon^{4}\right)
\end{aligned}
$$

Depending on where we decide to end the expansion. Hence

$$
\begin{aligned}
& v(x+\epsilon)-v(x-\epsilon) /(2 \epsilon)=v^{\prime}(x)+O\left(\epsilon^{2}\right) \quad \text { or } \\
& v(x+\epsilon)-v(x-\epsilon) /(2 \epsilon)=v^{\prime}(x)+\frac{\epsilon^{2}}{6} v^{\prime \prime \prime}(x)+O\left(\epsilon^{3}\right)
\end{aligned}
$$

First, choosing $v=p y^{\prime}, x=x_{j}$ and $\epsilon=\frac{h}{2}$, we get that

$$
\left(P y^{\prime}\right)^{\prime}\left(x_{j}\right)=\frac{\left[\left(p y^{\prime}\right)\left(x_{j}+\frac{h}{2}\right)-\left(p y^{\prime}\right)\left(x_{j}-\frac{h}{2}\right)\right]}{h}+O\left(h^{2}\right)
$$


Then, choosing $v=y, \epsilon=h / 2$, and $x=x_{j}+\frac{h}{2}$ and $x=x_{j}-\frac{h}{2}$, we get that

$$
\begin{aligned}
& y^{\prime}\left(x_{j}+\frac{h}{2}\right)=\frac{y\left(x_{j}+h\right)-y\left(x_{j}\right)}{h}-\frac{h^{2}}{24} y^{\prime \prime \prime}\left(x_{j}+\frac{h}{2}\right)+O\left(h^{3}\right) \\
& y^{\prime}\left(x_{j}-\frac{h}{2}\right)=\frac{y\left(x_{j}\right)-y\left(x_{j}-h\right)}{h}-\frac{h^{2}}{24} y^{\prime \prime \prime}\left(x_{j}-\frac{h}{2}\right)+O\left(h^{3}\right)
\end{aligned}
$$

Inserting these quantities, we obtain:

$$
\begin{aligned}
\left(p y^{\prime}\right)^{\prime}\left(x_{j}\right)=\frac{1}{h}\left[p\left(x_{j}+\frac{h}{2}\right) \frac{y\left(x_{j}+h\right)-y\left(x_{j}\right)}{h}-p\left(x_{j}-\frac{h}{2}\right) \frac{y\left(x_{j}\right)-y\left(x_{j}-h\right)}{h}\right] \\
+\frac{h}{24}\left[p\left(x_{j}+\frac{h}{2}\right) y^{\prime \prime \prime}\left(x_{j}+\frac{h}{2}\right)-p\left(x_{j}-\frac{h}{2}\right) y^{\prime \prime \prime}\left(x_{j}-\frac{h}{2}\right)\right]+O\left(h^{2}\right)
\end{aligned}
$$

Although the middle terms seems to only $O(h)$, we get by applying the Mean Value Theorem, that

$$
p\left(x_{j}+\frac{h}{2}\right) y^{\prime \prime \prime}\left(x_{j}+\frac{h}{2}\right)-p\left(x_{j}-\frac{h}{2}\right) y^{\prime \prime \prime}\left(x_{j}-\frac{h}{2}\right)=O(h)
$$

And so

$$
\left(p y^{\prime}\right)^{\prime}\left(x_{j}\right)=\frac{1}{h}\left[p\left(x_{j}+\frac{h}{2}\right) \frac{y\left(x_{j}+h\right)-y\left(x_{j}\right)}{h}-p\left(x_{j}-\frac{h}{2}\right) \frac{y\left(x_{j}\right)-y\left(x_{j}-h\right)}{h}\right]+O\left(h^{2}\right)
$$

Denoting $p\left(x_{j} \pm \frac{h}{2}\right)$ by $p_{j \pm 1 / 2}$, and omitting the $O\left(h^{2}\right)$ terms, we are led to the approximation:

$$
L_{h} y_{j}=\frac{1}{h^{2}}\left(-p_{j-\frac{1}{2}} y_{j-1}+\left[p_{j-\frac{1}{2}}+p_{j+\frac{1}{2}}+h^{2} q\left(x_{j}\right)\right] y_{j}-p_{j+\frac{1}{2}} y_{j+1}\right)
$$

And thus, to the difference equation becomes

$$
-p_{j-\frac{1}{2}} y_{j-1}+\left[p_{j-\frac{1}{2}}+p_{j+\frac{1}{2}}+h^{2} q\left(x_{j}\right)\right] y_{j}-p_{j+\frac{1}{2}} y_{j+1}=h^{2} f\left(x_{j}\right)
$$

We remark that although we have not kept careful track of the error terms, it is possible to show that the local truncation $\tau_{j}=L_{h} y\left(x_{j}\right)-L y\left(x_{j}\right)$ satisfies

$$
\left|\tau_{j}\right| \leq K h^{2}\left[\max _{[a, b]}\left|y^{(4)}(x)\right|+\max _{[a, b]}\left|\left(p y^{(3)}\right)^{\prime}(x)\right|\right]
$$

Where $K$ is a constant that does not depend on $y$ or $h$.

Now setting $a_{j}=p_{j-\frac{1}{2}}+p_{j+\frac{1}{2}}+h^{2} q\left(x_{j}\right)$, we obtain the linear system

$$
\left(\begin{array}{cccccc}
a_{1} & -p_{\frac{3}{2}} & 0 & 0 & \ldots & 0 \\
-p_{\frac{3}{2}} & a_{2} & -p_{\frac{5}{2}} & 0 & \ldots & 0 \\
\cdot & \cdot & \cdot \cdot & \cdot & \cdot & \cdot \\
\cdot & \cdot & \cdot & \cdot & \cdot & \cdot \\
0 & \cdot & & -p & a_{N-2} & -p_{N-\frac{3}{2}} \\
0 & \cdot & & & -p_{N-\frac{3}{2}} & a_{N-1}
\end{array}\right)\left[\begin{array}{c}
y_{1} \\
y_{2} \\
\cdot \\
\cdot \\
y_{N-2} \\
y_{N-1}
\end{array}\right]=h^{2}\left[\begin{array}{c}
f\left(x_{1}\right) \\
f\left(x_{2}\right) \\
\cdot \\
\cdot \\
f\left(x_{N-2}\right) \\
f\left(x_{N-1}\right)
\end{array}\right]+\left[\begin{array}{c}
p_{\frac{1}{2}} y_{a} \\
0 \\
\cdot \\
\cdot \\
0 \\
p_{N-\frac{1}{2}} y_{b}
\end{array}\right]
$$

Theorem 3.1. Assume that $q(x), r(x)$ are an element of the space real-valued continuous function on $[a, b]$ and $q \geq 0$. Then the boundary value problem for the linear differential equation

$$
-y^{\prime \prime}+q(x) y=r(x) \text { on }[a, b]
$$

with homogeneous boundary conditions

$$
y(a)=y(b)=0
$$

has a unique solution $y \in c^{2}[a, b]$.

Now for the numerical solution of the differential equation (11) we choose an equidistance grid $x_{j}=a+j h, j=$ $1,2, \ldots, n+1$ with the step size $h=(b-a) / n+1$ and $n \in N$ at the internal grid points $x_{j}, j=1,2, \ldots, n$, we replace the differential equation by the difference quotient 


$$
y^{\prime \prime}\left(x_{j}\right) \approx \frac{1}{h^{2}}\left[y\left(x_{j+1}\right)-2 y\left(x_{j}\right)+y\left(x_{j-1}\right)\right]
$$

and substituting into equation (11) we obtain the system of equations

$$
-\frac{1}{h^{2}}\left[y_{j-1}-\left(2+h^{2} q_{j}\right) y_{j}+y_{j+1}\right]=r_{j}, j=1,2, \ldots, n
$$

For approximate values $y_{j}$ to the exact solution $y\left(x_{j}\right)$. Here we have set $q_{j}=q\left(x_{j}\right)$ and $r_{j}=r\left(x_{j}\right)$. The system has to be complemented by the two boundary conditions

$$
y_{0}=y_{n+1}=0
$$

For an abbreviated notation we introduce the $n \times n$ tri-diagonal matrix. Let

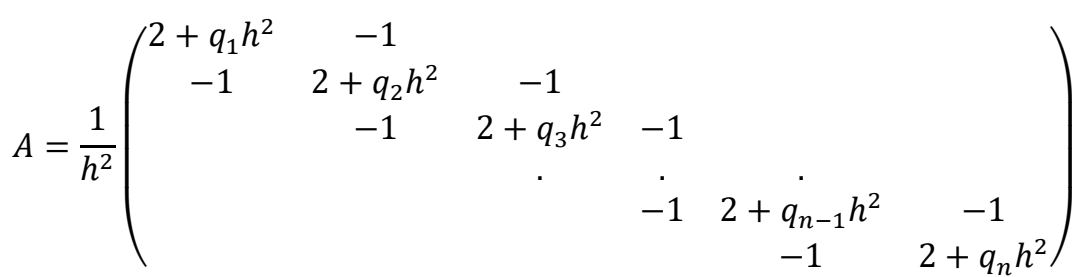

And the vectors $Y=\left(y_{1}, \ldots, y_{n}\right)^{T}$ and $R=\left(r_{1}, \ldots, r_{n}\right)^{T}$. Then our system of equations including the boundary conditions

$$
A Y=R
$$

This tri-diagonal linear system is non-singular and symmetric can be easily solved for $Y$ from any right-hand side of $R$.

Theorem 3.2. For each $h>0$ the difference equations (14) with (15) have a unique solution.

\section{Stability and Convergence Analysis}

The error and convergence analysis are initiated by first establishing the following two lemmas.

Lemma 4.1. Denote by $A$ the matrix of the finite difference method for $q \geq 0$ and by $A_{0}$ the corresponding matrix for $q=0$.

$$
A_{0}=\left[\begin{array}{cccccc}
2 & -1 & 0 & . & . & 0 \\
-1 & 2 & -1 & 0 & . & 0 \\
0 & -1 & 2 & -1 & . & \cdot \\
. & \cdot & \cdot & \cdot & . & \cdot \\
0 & \cdot & \cdot & -1 & 2 & -1 \\
0 & \cdot & . & . & -1 & 2
\end{array}\right]
$$

Then $0 \leq A^{-1} \leq A_{0}^{-1}$.

That is, all the components of $A^{-1}$ are nonnegative and smaller than or equal to the corresponding components of $A_{0}{ }^{-1}$.

Proof: The columns of the inverse $A^{-1}=\left(a_{1}, a_{2}, \ldots, a_{n}\right)$ satisfy $A a_{j}=e_{j}$ for $j=1, \ldots, n$ with the canonical unit vectors $e_{1}, e_{2}, \ldots, e_{n}$ in $\mathfrak{R}^{n}$. The Jacobi iterations for the solution of $A z=e_{j}$ starting with $z_{0}=0$ are given by

$$
z_{n+1}=-D^{-1}\left(A_{L}+A_{R}\right) z_{n}+D^{-1} e_{j}, n=0,1, \ldots
$$

With the usual splitting $A=D+A_{L}+A_{R}$ of $A$ into its diagonal, lower and upper triangular parts. Since the entries of $D^{-1}$ and $-D^{-1}\left(A_{L}+A_{R}\right)$ are all nonnegative, it follows that $A^{-1} \geq 0$. Analogously, the iterations

$$
z_{n+1}=-D^{-1}\left(A_{L}+A_{R}\right) z_{n}+D^{-1} e_{j}, n=0,1, \ldots
$$

yields the columns of $A_{0}^{-1}$. Therefore, from $D_{0}{ }^{-1} \geq D^{-1}$ we conclude that $A_{0}^{-1} \geq A^{-1}$.

Lemma 4.2. Assume that $y$ is an element of space real-valued function and four times continuously differentiable. 
Then

For all $x \in[a+h, b-h]$.

$$
\left|y^{\prime \prime}(x)-\frac{1}{h^{2}}[y(x+h)-2 y(x)+y(x-h)]\right| \leq \frac{h^{2}}{12}\left\|y^{(4)}\right\|_{\infty}
$$

Theorem 4.1. Assume that the solution to the boundary value problem Eq. (11) with (12) is four times continuously differentiable. Then the error of the finite difference approximation can be estimated by

$$
\left|y\left(x_{j}\right)-y_{j}\right| \leq \frac{h^{2}}{96}\left\|y^{(4)}\right\|_{\infty}(b-a)^{2}, \quad j=1, \ldots, n
$$

Proof: by lemma (4.2) the local truncation error

We have the estimate

$$
z_{j}=y^{\prime \prime}\left(x_{j}\right)-\frac{1}{h^{2}}\left[y\left(x_{j+1}\right)-2 y\left(x_{j}\right)+y\left(x_{j-1}\right)\right]
$$

$$
\left|z_{j}\right| \leq \frac{h^{2}}{12}\left\|y^{(4)}\right\|_{\infty}, j=1, \ldots, n
$$

Since

$$
-\frac{1}{h^{2}}\left[y\left(x_{j+1}\right)-\left(2+h^{2} q_{j}\right) y\left(x_{j}\right)+y\left(x_{j-1}\right)\right]=-y^{\prime \prime}\left(x_{j}\right)+q_{j} y\left(x_{j}\right)+z_{j}=r_{j}+z_{j}
$$

The vector $\bar{y}=\left(y\left(x_{1}\right), \ldots, y\left(x_{n}\right)\right)^{T}$ given by the exact solution solves the linear system

$$
A \bar{Y}=R+Z
$$

Where $Z=\left(z_{1}, z_{2}, \ldots, z_{n}\right)^{T}$. Therefore,

$$
A(\bar{Y}-Y)=Z \text {, }
$$

and from this, using lemma (4.1) and the estimate (17), we obtain

$$
\left|y\left(x_{j}\right)-y_{j}\right| \leq\left\|A^{-1} Z\right\|_{\infty} \leq \frac{h^{2}}{12}\left\|y^{(4)}\right\|_{\infty}\left\|A_{0}^{-1} e\right\|_{\infty}, j=1, \ldots, n
$$

Where $e=(1, \ldots, 1)^{T} . A_{0}^{-1} e$ obtained by the boundary value problem of the type

$$
-y_{0}{ }^{\prime \prime}=1, y_{0}(a)=y_{0}(b)=0
$$

Has the exact solution $y_{0}(x)=\frac{1}{2}(x-a)(b-x)$.

Since $y_{0}=0$, in this case, as a consequence of (18) the finite difference approximation coincides with the exact solution i.e. $e=A_{0} Y=A_{0} \bar{Y}$.

Hence

$$
\left\|A_{0}^{-1} e\right\|_{\infty} \leq\left\|y_{0}\right\|_{\infty}=\frac{1}{8}(b-a)^{2}, j=1, \ldots, n
$$

Inserting this into Eq. (18) completes the proof.

\section{Numerical Examples}

To validate the applicability of the method, we have considered the following model problems with exact solution. For each $N$, the point wise absolute errors are approximated by the formula, $\left|e_{j}\right|=\left|y\left(x_{j}\right)-y_{j}\right|$ for $j=0,1, \ldots, N$, where $y\left(x_{j}\right)$ and $y_{j}$ are the exact and computed approximate solution of the given problem respectively, at the nodal point $x_{j}$.

Example 1: Consider the boundary value problem

$$
y^{\prime \prime}=x+y
$$

with the boundary conditions $y(0)=0, y(1)=0$. The exact solution for this problem is $y(x)=\frac{\sinh x}{\sinh 1}-x$.

The numerical solutions in terms of point wise absolute errors by comparing with the exact solutions are given in Table 1 . 
Table 1. The comparison of approximate solution with exact solution for Example 1 at different values of the step size $h$

\begin{tabular}{c|ccc|c}
\hline Mesh size h & $\boldsymbol{x}_{\boldsymbol{j}}$ & Computed value & Exact value & Error \\
\hline \multirow{3}{*}{$\mathbf{0 . 2 5}$} & 0.25 & -0.03488 & -0.03505 & 0.00017 \\
& 0.5 & -0.05632 & -0.05659 & 0.00027 \\
& 0.75 & -0.05003 & -0.05028 & 0.00025 \\
\hline \multirow{3}{*}{$\mathbf{0 . 2}$} & 0.2 & -0.02855 & -0.02679 & 0.00176 \\
& 0.4 & -0.05028 & -0.05048 & 0.00034 \\
& 0.6 & -0.05804 & -0.05826 & 0.00022 \\
\hline
\end{tabular}

Figure 1. Comparison of the numerical with the exact solution for Example 1 at (a) $h=0.25$ and (b) $h=0.2$
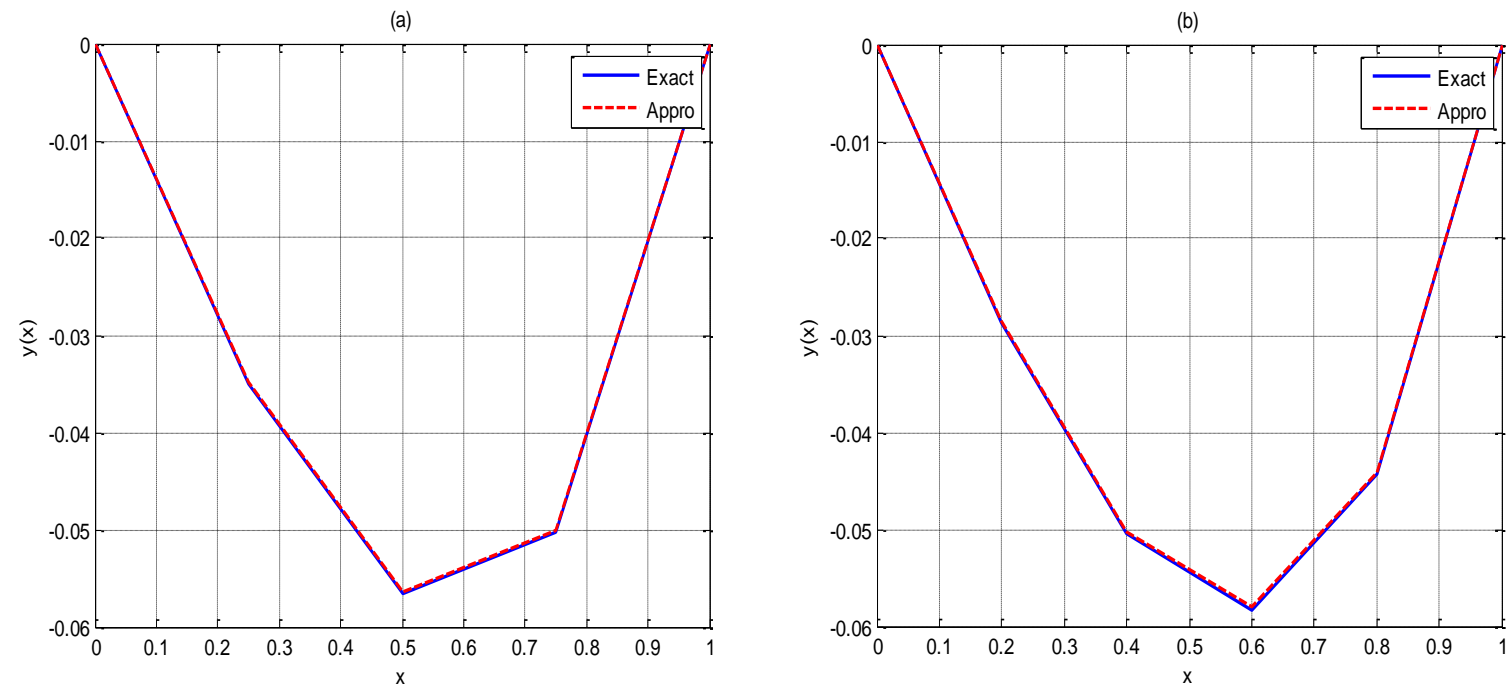

Example 2: Consider the boundary value problem

$$
y^{\prime \prime}+5 y^{\prime}+4 y=1,0 \leq x \leq 1
$$

with the boundary conditions: $y(0)=0, y(1)=0$, the exact solution is given by:

$$
y(x)=A e^{-x}+B e^{-4 x}+0.25, \text { where } A=\frac{e^{-3}-e}{4\left(1-e^{-3}\right)}, B=-0.25-A
$$

The numerical solutions by comparing with the exact solutions are given in Table 2.

Table 2. The comparison of approximate solution with exact solution for Example 2 at $h=0.25$.

\begin{tabular}{cccc}
\hline $\boldsymbol{x}_{\boldsymbol{i}}$ & Computed value & Exact value & Error \\
\hline $\mathbf{0 . 2 5}$ & -0.15374 & -0.13047 & 0.02327 \\
$\mathbf{0 . 5}$ & -0.12711 & -0.11465 & 0.01246 \\
$\mathbf{0 . 7 5}$ & -0.06295 & -0.05913 & 0.00382 \\
\hline
\end{tabular}


Figure 2. Comparison of the numerical with the exact solution for Example 2 at $h=0.25$.

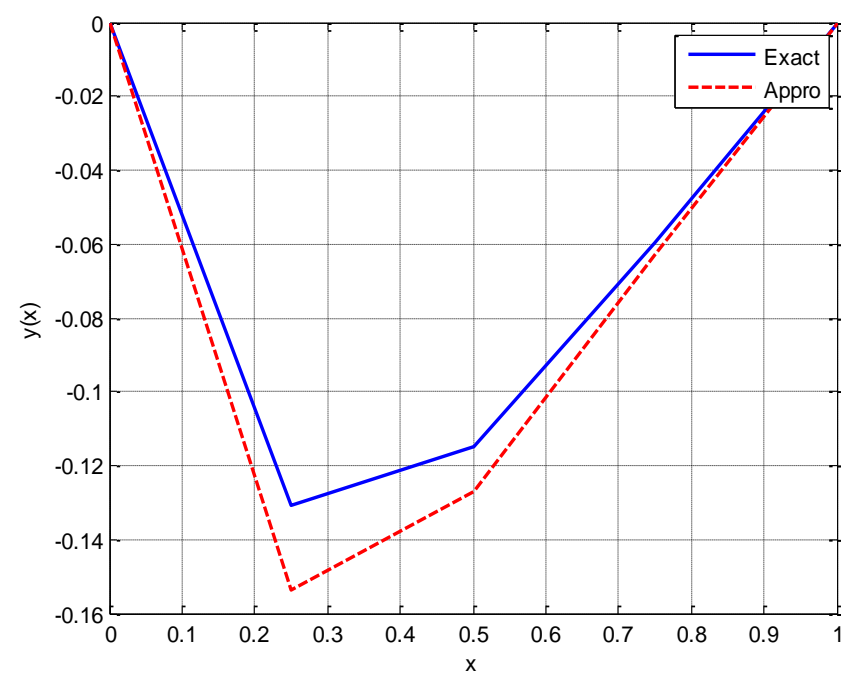

\section{Applications}

Problem 1: A steady one-dimensional heat transfer problem consists of heat conduction along a constant area of rod with heat convection to the surrounding. The ends of the rod maintained at the constant temperature $T_{1}$ and $T_{2}$. An energy balance on a differential element of the rod yields the following second order boundary value problem of ordinary differential equation

$$
\begin{aligned}
& T^{\prime \prime}-\alpha^{2} T=-\alpha^{2} T_{a} \\
& T\left(x_{1}\right)=T(0)=T_{1} \\
& T\left(x_{2}\right)=T(L)=T_{2}
\end{aligned}
$$

Where $T$ is the temperature of the $\operatorname{rod}(\mathrm{c})$ and $\alpha^{2}=h p / k A\left(\mathrm{~cm}^{-2}\right)$ where $h$ is heat transfer coefficient $\left(\mathrm{J} / \mathrm{s}-\mathrm{m}^{2}-\mathrm{k}\right)$, $p$ is perimeter of the $\operatorname{rod}(m), k$ is the thermal conductivity, $A$ is surface area of the $\operatorname{rod}\left(m^{2}\right)$ and $T_{a}$ is temperature of surrounding $\left({ }^{\circ} \mathrm{C}\right)$.

Heat transfer by convection is governed by Newton's Law of cooling: $q_{c}(x)=h A\left(T-T_{a}\right)$

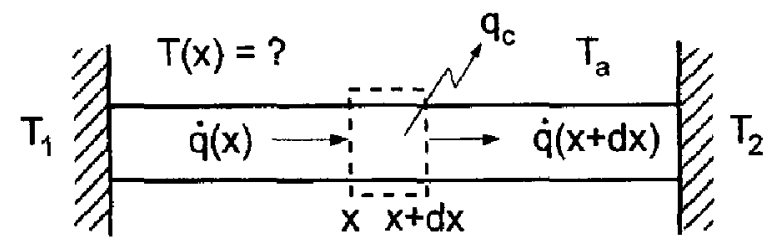

Figure 6.1: Steady one-dimensional heat transfer in a rod.

Now the general solution of equation (19) is

$$
T(x)=A e^{\alpha x}+B e^{-\alpha x}+T_{a}
$$

We can demonstrate by direct substitution the boundary conditions into the general solution

$$
A=\frac{\left(T_{2}-T_{a}\right)-\left(T_{1}-T_{2}\right) e^{-\alpha L}}{e^{\alpha L}-e^{-\alpha L}} \text { and } B=\frac{\left(T_{1}-T_{2}\right) e^{-\alpha L}-\left(T_{2}-T_{a}\right)}{e^{\alpha L}-e^{-\alpha L}}
$$

Consider a $\operatorname{rod} L=1 \mathrm{~cm}$ long with $T(0)=0^{\circ} \mathrm{C}$ and $T(1)=100^{\circ} \mathrm{C}$. Let $\alpha^{2}=16 \mathrm{~cm}^{-2}$ and $T_{a}=0{ }^{\circ} \mathrm{C}$ for these conditions the general equation yields

$$
T(x)=1.832179\left(e^{4 x}-e^{-4 x}\right)
$$

Let us solve the heat transfer by using the second order finite difference method

$$
T^{\prime \prime}-\alpha^{2} T=-\alpha^{2} T_{a} \text { and } T(0)=0^{\circ} \mathrm{C}, T(1)=100^{\circ} \mathrm{C}
$$


Replace $T^{\prime \prime}$ by second order central difference approximation and evaluating all the terms at the grid points $i$ gives

$$
\frac{T_{i+1}-2 T_{i}+T_{i-1}}{\Delta x^{2}}+O\left(\Delta x^{2}\right)-\alpha^{2} T_{i}=-\alpha^{2} T_{a}
$$

Multiplying by $\Delta x^{2}$ and truncating the remainder yields

$$
T_{i-1}-\left(2+\alpha^{2} \Delta x^{2}\right) T_{i}+T_{i+1}=-\alpha^{2} \Delta x^{2} T_{a}
$$

Let $\alpha^{2}=16 \mathrm{~cm}^{-2}$ and $T_{a}=0^{\circ} \mathrm{C}, \Delta x=0.25$. Then $T_{i-1}-3 T_{i}+T_{i+1}=0$

Applying the three grid points $x=0.25,0.5,0.75$

$$
\begin{aligned}
& x=0.25, \quad T_{1}-3 T_{2}+T_{3}=0, T_{1}=0 \\
& x=0.5, \quad T_{2}-3 T_{3}+T_{4}=0 \\
& x=0.75, \quad T_{3}-3 T_{4}+T_{5}=0, \quad T_{5}=100
\end{aligned}
$$

We get the following tri-diagonal system of finite difference equations

$$
\left[\begin{array}{ccc}
-3 & 1 & 0 \\
1 & -3 & 1 \\
0 & 1 & -3
\end{array}\right]\left[\begin{array}{l}
\mathrm{T}_{2} \\
\mathrm{~T}_{3} \\
\mathrm{~T}_{4}
\end{array}\right]=\left[\begin{array}{c}
0 \\
0 \\
-100
\end{array}\right]
$$

By Gauss elimination we get

$$
\mathrm{T}_{2}=4.7618, \mathrm{~T}_{3}=14.2857, \mathrm{~T}_{4}=38.0952 .
$$

\section{Conclusion}

The accuracy of finite difference method for second order linear Boundary value problem in ODEs depends on the size of the sub-interval $h$ and also on the order of approximation. As we reduce $h$, the accuracy improves but the number of equations to be solved also increases. When a large number of subdivisions are used, we do not treat a difference equation as a set of simultaneous equations. As a result, the amount of computation time required may become excessive, and good accuracy may be difficult to achieve. The use of higher-order approximations will yield greater accuracy for the same mesh size but results in considerable complication, especially near the end points of the interval. In practice, it is advisable to solve the linear system for several different values of $h$. A comparison of the solutions at the same mesh points indicate the more accuracy being obtained.

\section{References}

[1]. Badradeen A., Mohsin H. A. Hashim (2014), “Shooting method in solving Boundary Value Problem”, IJRRAS 21(1).

[2]. Bernard Bialecki (1991), "Sinc-Collection Methods for Two-Point Boundary Value Problems", IMA Journal of Numerical Analysis, Volume 11, Issue 3, Pages 357-375.

[3]. Colletz L. (1996), "The Numerical Treatment of Differential Equations”, $3^{\text {rd }}$ edition, Springer-Verlag, Berlin, 241-260.

[4]. H.B. Keller, "Numerical Solution of Two-Point Boundary Value Problems", SIAM, Philadelphia, fourth printing, 1990.

[5]. Henrici, P., Discrete variable methods in ordinary differential equations, New York: Wiley 1964.

[6]. Ikram A. T. (2006), Nonpolynomial spline approach to the solution of a system of second-order boundary value problems, Applied Mathematics and computation 173(2), pp. 1208-1218.

[7]. Iyenger, S. R. K., Jain, R. K., "Numerical Methods. New age international publishers”, New Delhi, 2009.

[8]. Joe D. Hoffmen, "Numerical Methods for Engineers and Scientists", McGrawHill, New York, 1992.

[9]. Lakshmi R., Muthuselvi M. (2013), "Numerical solutions for boundary value problem using finite difference method", IJIRSET 2: 5305-5313.

[10]. Mathews J.H. (1999), "Numerical methods using MATLAB", 4e, Upper Saddle River.

[11]. Randalls J. LeVeque, "Finite Difference Methods for Ordinary and Partial Differential Equations", Philadelphia, Washington, 2007.

[12]. Roark, A. L., Shampine, L. F., "On the numerical solution of a linear two-point boundary Value problem", Sandia Laboratory Technical Memorandum SC-TM-67-588. Albuquerque, New Mexico, 1967.

[13]. Shampine, L. F.: "Boundary value problems for Ordinary Differential Equations", SIAM J. Numer. Anal.5, 219-242 (1968).

[14]. Srivastava, P. K., Kumar, M., Mohapatra, R. N., (2011), Quinitic nonpolynomial spline method for the solution of a second-order boundary-value problem with engineering applications, computers and 
Mathematics with Applications, V62(4), pp 1707-1714.

[15]. U.M. Ascher, R.M.M. Mattheij and R.D. Russell, "Numerical Solution of Boundary Value Problems for Ordinary Differential Equations", Corrected reprint of the 1988 original, Classics in Applied Mathematics, 13, SIAM, Philadelphia, 1995.

[16]. Zahra, W. K. (2004), Numerical solutions for boundary value problems for ordinary differential equations by using spline functions, MSc. Thesis, Faculty of Engineering, Tanta University, Egypt. 